\title{
The Influence of the Transverse Sensitivities of Vibration Force Sensors on the Efficiency of an Active Vibration Protection System
}

\author{
Aleksey Vladimirovich Kirjukhin, Oleg Osherevich Milman, Anton Viktorovich Ptakhin \\ Kaluga State University KE Tsiolkovsky, St. Razina Street, 26, Kaluga, 248000, Russia \\ SPC Turbocon, Komsomolskaya Rosha Street, 43, Kaluga, 248010, Russia
}

\begin{abstract}
The article considers the force sensors (FS) transverse sensitivity influence on the efficiency of active vibration protection systems (AVS) with control by disturbance, in the feedback of which vibrational force sensors are used. The errors of force measurement by sensors used in different conditions are calculated. The AVS efficiency determined from force sensors has been compared with the AVS efficiency determined by the resultant effects on the foundation. The calculated and experimental values of the AVS efficiency are given under the conditions when three-component forces act on the sensors and when transverse sensitivities are present in the sensors.
\end{abstract}

Key words: active vibration protection system, vibrational force, force sensor, transverse sensitivity, transfer function, feedback.

\section{INTRODUCTION}

To reduce the dynamic forces transmitted from vibroactive installations of various purposes to attached structures (foundation), both passive vibration protection (shock absorption) and active vibration protection systems (AVS) can be used. Among numerous studies considering various AVS schemes, the systems, in the feedback loops of which vibration (dynamic) force sensors are used [1-4] occupy an important place. Force measurement and its active compensation are carried out in one direction. It is believed that the sensors measure only forces in the direction of the AVS action, i.e., they have zero transverse sensitivities. However, the vibration of real installations usually has a spatial (three-component) character. Therefore, sensors, in the general case, are affected by spatial vibrational forces having nonzero components in three mutually perpendicular directions. Moreover, even single-component force sensors of leading manufacturers have non-zero transverse sensitivities. Three-component force sensors also have cross (transverse) sensitivities. For example, in the passport to the type 261A13 force sensors produced by the company PCB, transverse sensitivities are indicated to be up to $20 \%$ of axial values.
Therefore, the sensors respond to disturbances both in the direction of the measurement axis and to the transverse components of the force. With the actual zero force along the measured axis, in the case of the transverse components, presence acting on the sensor, the signal along this axis from the sensor will not be zero. This leads to errors in the force measurement, and in the case of their use in the AVS, it can affect its effectiveness. The paper considers the calculated effect of the force sensors transverse sensitivities on the AVS efficiency with such sensors in its feedback loop, with verification at the test bench of a three-component broadband active damping of vibrational forces transmitted to the foundation. In addition, the presence of transverse components of the sensors sensitivity inevitably leads to cross-connections in the control system. In [5], it is noted that the synthesis of controllers in multidimensional systems is extremely difficult, since one of the main problems for such systems is the problem of "decoupling" control channels.

\section{CALCULATION OF IMPACT OF THE FORCE SENSOR TRANSVERSE SENSITIVITY ON THE FORCE MEASUREMENT ERROR}

A single-mass system is considered in Figure 1 which vibrates in three directions $\mathrm{x}, \mathrm{y}, \mathrm{z}$ under the influence of forces $\mathrm{Qx}$, Qy and Qz. Mass $M$ is set on vibration isolators and three-component force sensor with complex vibration rigidity $\mathrm{Cx}, \mathrm{Cy}, \mathrm{Cz}$ and $\mathrm{Cdx}, \mathrm{Cdy}, \mathrm{Cdz}$, respectively. Oscillations of the mass through vibration isolators act on force sensors rigidly fixed to the foundation under vibration isolators. To reduce vibrational forces transferred to the foundation, AVS with control by disturbance acts in each direction. AVS feedback loops use force sensors that have nonzero cross (transverse) sensitivities. The same sensors measure the resulting force acting on the foundation in each direction.

To implement active damping in the $\mathrm{z}$ direction, the signal component from the force sensors along the $\mathrm{z}$ axis is fed to the AVS with the transfer function $\mathrm{Hz}$ and gain kz. The actuator (vibrator) of the AVS acts on the mass $\mathrm{M}$ in the $\mathrm{z}$ direction by the active compensating force Qaz . AVS schemes for $\mathrm{x}$ and $\mathrm{y}$ directions are similar. To control the resulting signals, a spectrum analyzer is used. 

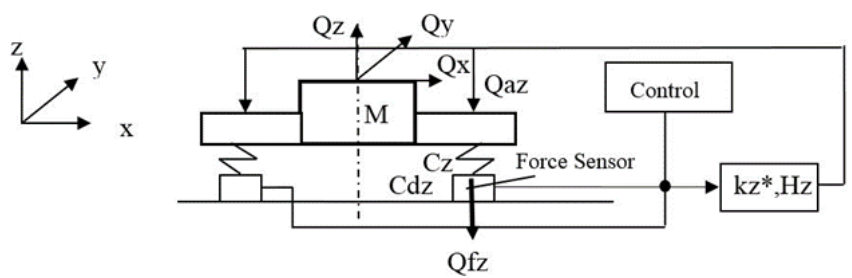

Figure 1: The complex transverse sensitivity coefficients of the force sensor will be denoted

$$
A i j=\frac{Q^{\prime} i j}{Q^{\prime} j}
$$

where $Q^{\prime} i j$ is the force measured by the sensor in direction $i$ from the action on the sensor in direction $j$ of dynamic force $Q^{\prime} j$ in the absence of forces in other directions. Taking into account the accepted notation, the expressions for the force $Q f z$ acting in the z direction on the foundation and the force $Q d z$ measured by the sensor during the oscillations $\mathrm{x}$ of the mass $\mathrm{M}$ in three directions can be

written as

$$
\begin{gathered}
Q f z=C z * Z \\
Q d z=C z * Z+A z x * C x * X+A z y * C y * Y
\end{gathered}
$$

where $\mathrm{X}, \mathrm{Y}, \mathrm{Z}$ are the displacements of the mass $\mathrm{M}$ in the directions $\mathrm{x}, \mathrm{y}, \mathrm{z}$, respectively, $\mathrm{Cx}, \mathrm{Cy}, \mathrm{Cz}$ are the complex vibrational rigidity of the vibration isolators. Expressions for forces in two other directions are written similarly. From this formula it follows that when there is the three-component oscillation of mass $\mathrm{M}$ and the non-zero transverse sensitivity of the sensor, the sensor measures the force not actually exerted on the foundation $Q f z=C z^{*} Z$, but a force resulting from the transverse sensitivity of the sensor, the sum of forces consisting of three components (3) .

Let us estimate for different versions of the AVS operation the sensor measurement errors of the forces acting on the foundation. Let us assume that the parameters of all sensors in the directions are identical. Then the equation (3) for the force measured by the sensors in the $\mathrm{z}$ direction can be written as

$$
Q d z=Q f z *\left(1+A z x * \frac{Q^{\prime} x}{Q^{\prime} z}+A z y * \frac{Q^{\prime} y}{Q^{\prime} z}\right)
$$

where $Q f z=Q^{\prime} z=C z^{*} Z$ is the force acting on the foundation equal to the force acting on the sensors in the direction $\mathrm{z} ; Q^{\prime} x=C x^{*} X$ force acting on the sensors in the x direction; $Q^{\prime} y=C y^{*} Y$ is the force acting on the sensors in the y direction. The obtained formula shows that in the presence of transverse sensitivities of a force sensor, the error in measuring the force acting on the foundation depends on the transverse sensitivity coefficients and on the magnitude of the forces acting on the sensor in three directions.

\section{CALCULATION OF IMPACT OF THE FORCE SENSOR TRANSVERSE SENSITIVITIES ON THE AVS EFFICIENCY}

Let us consider the error in measuring forces by the sensor in three cases.

1.All three AVS channels are turned off.

Let the forces acting on the sensor $Q^{\prime} j$ be the same in three directions, and the transverse sensitivity coefficients equal $A i j$ $= \pm 0.2$. Then, depending on the phase relationship between the coefficients, the force measured by the sensor is

$$
Q d z=Q f z *(1 \pm A z x \pm A z y)=\left(\begin{array}{c}
1.4 * Q f z \\
Q f z \\
-0.6 * Q f z
\end{array}\right)
$$

Its difference from the force actually acting on the foundation can reach $40 \%$. With large transverse forces acting on the force sensor and a small force in the $\mathrm{z}$ direction, the measurement error may be greater.

2. One AVS is turned on in the $\mathrm{z}$ direction.

When AVS is working in the z direction, the force $Q^{\prime} z$ in this direction decreases, with constant transverse forces $Q^{\prime} x$ and $Q^{\prime} y$, and therefore, according to (4), the measurement error can increase.

3. Three AVS are turned on in the $\mathrm{x}, \mathrm{y}, \mathrm{z}$ directions.

With the equally efficient operation of all three AVSs, forces in all three directions are equally reduced, and therefore, according to (4), the measurement error can be commensurate with the error when the AVSs are off. The estimates show that when using AVS in one direction sensor measurement error of the force acting on the foundation in the direction of AVS action may increase substantially in comparison with the case when the AVS is off, and if the AVS works in three directions it can be commensurate with the error when the AVS is off.

AVS in each direction $i=x, y, z$ forms an active compensating force $Q a i$ based on the measured by the sensor forces Qdi. Using (3), the expression for the active force $Q a z$ in the z direction can be written as 
Aleksey Vladimirovich Kirjukhin et al., International Journal of Emerging Trends in Engineering Research, 8(1), January 2020,203 - 207

$$
Q a z=k z * H z * Q d z=k z * H z * C z * Z *\left[1+\frac{(A z x * C x) * X}{C z * Z}+\frac{(A z y * C y) * Y}{C z * Z}\right]
$$

Active forces in the $\mathrm{y}$ and $\mathrm{z}$ directions are written by analogy. It is obvious (6) that the presence in the formula of the active force of the sensor transverse sensitivity coefficients Aij can affect the AVS efficiency. The effect on the mass $\mathrm{M}$ of the active forces created by the AVS leads to both a change in the forces measured by the sensors and the forces actually acting on the foundation. These changes may be different when vibration is excited in more than one direction (6), therefore, we introduce two AVS efficiencies. Efficiency by force sensors

$$
\eta_{d i}=20 * \lg \left(\frac{|Q \operatorname{di}(k z=0)|}{|Q d i(k z \neq 0)|}\right)
$$

and efficiency by actual force acting on the foundation

$$
\eta_{f i}=20 * \lg \left(\frac{|Q f i(k z=0)|}{|Q f i(k z \neq 0)|}\right)
$$

\section{THE EXPERIMENT AND CALCULATIONS RESULTS}

At a special test bench, the effect of the transverse sensitivities of force sensors on the AVS efficiency by force sensors was experimentally verified. An amortized plate was installed with a natural frequency of $30 \mathrm{~Hz}$ on four rubber-metal vibration isolators mounted on three-component force sensors type 261A13 manufactured by PCB. Plate vibration was excited by close-level broadband forces in the $\mathrm{x}, \mathrm{y}$, and $\mathrm{z}$ directions. The efficiency was measured using force sensors during the operation of AVS in the vertical direction $\mathrm{z}$ with the AVS channels turned off and on in the transverse directions $\mathrm{x}$ and $\mathrm{y}$, which provided damping of forces in these directions. In all three AVS channels, a resonant element with a frequency of 285 $\mathrm{Hz}$ with a transfer function was used as a regulator $\mathrm{H}=\left(\cos (\varphi) *(\mathrm{~s}+\gamma)-\omega^{*} \sin (\varphi)\right) /\left((\mathrm{s}+\gamma)^{\wedge} 2+\omega^{\wedge} 2\right)$ where $\gamma=0.8$ is the quality factor of the element; $\omega=2 * \pi * \mathrm{f}$ is the circular frequency; $\mathrm{f}=285 \mathrm{~Hz}$ is the resonator frequency, $\varphi=0$ is the correction phase; $s$ is the Laplace operator.

Figure 2 shows the graphs of measured efficiencies $\eta_{d z}$ in the direction $\mathrm{z}$ at the switched off 1 and switched on 2 AVS channels in transverse directions $x$ and $y$. When the AVS is turned on in the transverse directions $\mathrm{x}$ and $\mathrm{y}$ and, as a result, the transverse forces acting on the sensor are reduced, the efficiency $\eta_{d z}$ in the $\mathrm{z}$ direction increases by $8 \mathrm{~dB}(2.5$ times). This confirms the influence of the transverse sensitivity of the force sensors on the AVS efficiency by force sensors.

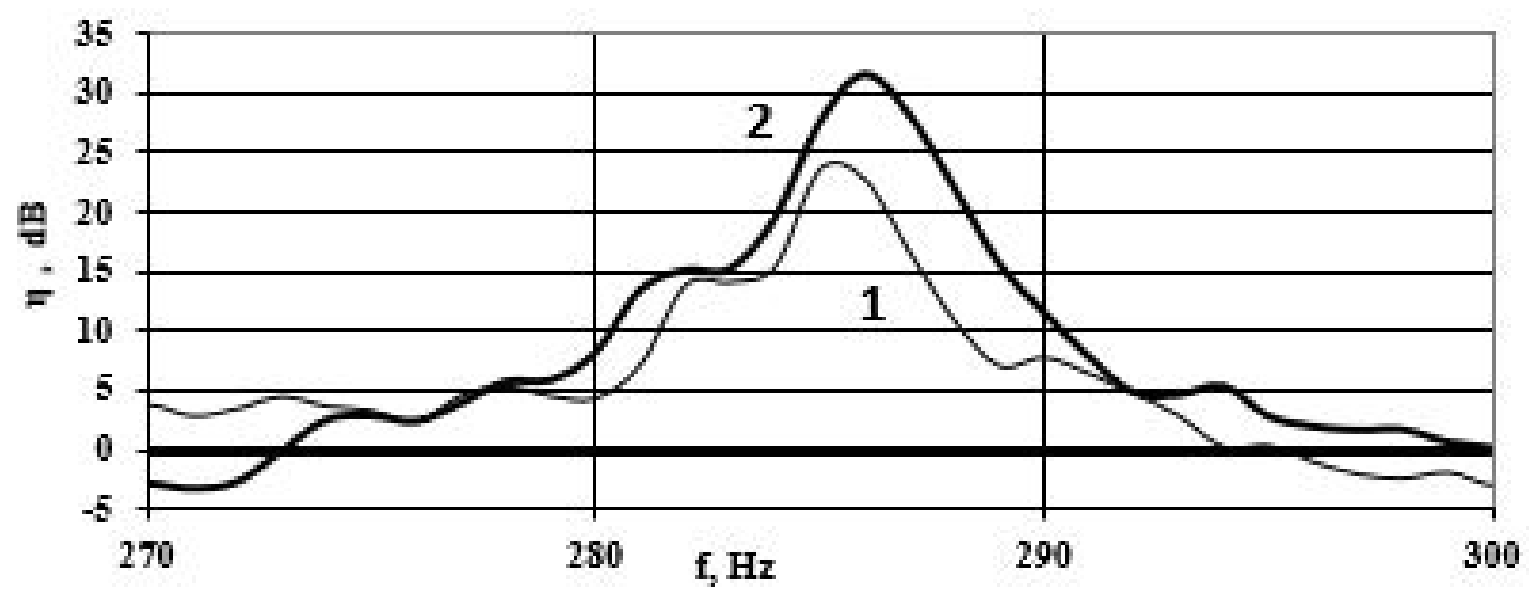

Figure 2: Experiment efficiency $\eta_{d z}$ by AVS force sensors with the resonant element $\mathrm{f}$ res $=285 \mathrm{~Hz}$ in the direction z. 1- only AVS channel $\mathrm{z}$ is in operation; 2- all three AVS channels $\mathrm{x}, \mathrm{y}, \mathrm{z}$ are in operation.

The purpose of the AVS is to reduce the forces acting on the foundation, so of interest is its efficiency by the foundation. Using force sensors, only AVS efficiency can be measured experimentally by sensors. The influence of the transverse sensitivities of the sensors on the AVS efficiency

by the foundation $\eta_{f z}$ and its comparison with the efficiency by the force sensors $\eta_{d z}$ was carried out by calculation. The calculation was performed on the 
mathematical model of the test bench for the same excitation forces along the $\mathrm{x}, \mathrm{y}, \mathrm{z}$ axes with the same transverse sensitivity coefficients of the force sensors Aij $=0,2$. Figure 3 shows a comparison of the experimental dependence
(1) $Q d z(f)$ with the calculated one (2) with the absence of active damping. Their good agreement was obtained up to frequencies of the order of $300 \mathrm{~Hz}$.

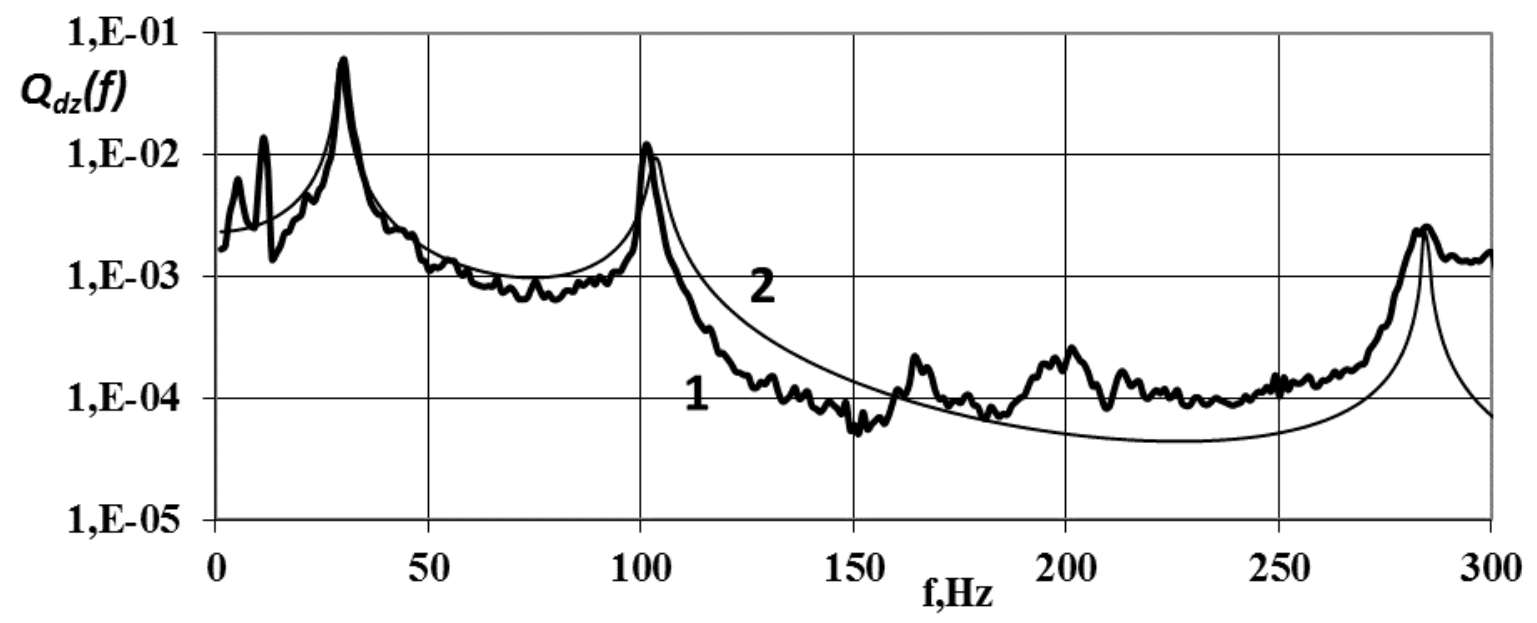

Figure 3: Comparison of the experimental dependence (1) of the force on the sensors $Q d z(f)$ from the frequency $f$ with the calculated one (2) in the $\mathrm{z}$ direction upon excitation by a three-component force.

Figure 4 shows the calculated efficiencies for the force sensors $\eta_{d z}$ and for the AVS foundation $\eta_{f z}$ in the $\mathrm{z}$ direction with the AVS turned off and on in the transverse directions with the same regulator in the form of a resonant element. The calculated influence of transverse forces on the efficiency of the sensors $\eta_{d z}$ is close to the experimentally measured in Figure 2 and relatively small, of the order of $6 \mathrm{~dB}$ (about two times). The influence of transverse forces on the $\eta_{f z}$ efficiency by the foundation is much greater. When the AVS are turned off in the transverse directions (when the transverse forces are applied to the sensor), the efficiency $\eta_{f z}$ is about $10 \mathrm{~dB}$. When they are turned on (when the transverse forces are reduced) it increases sharply (by $30 \mathrm{~dB}$, i.e. 30 times), and gets close to the efficiency by sensors $\eta_{d z}$, somewhat exceeding it (in this case by $6 \mathrm{~dB}$, i.e. in two times).

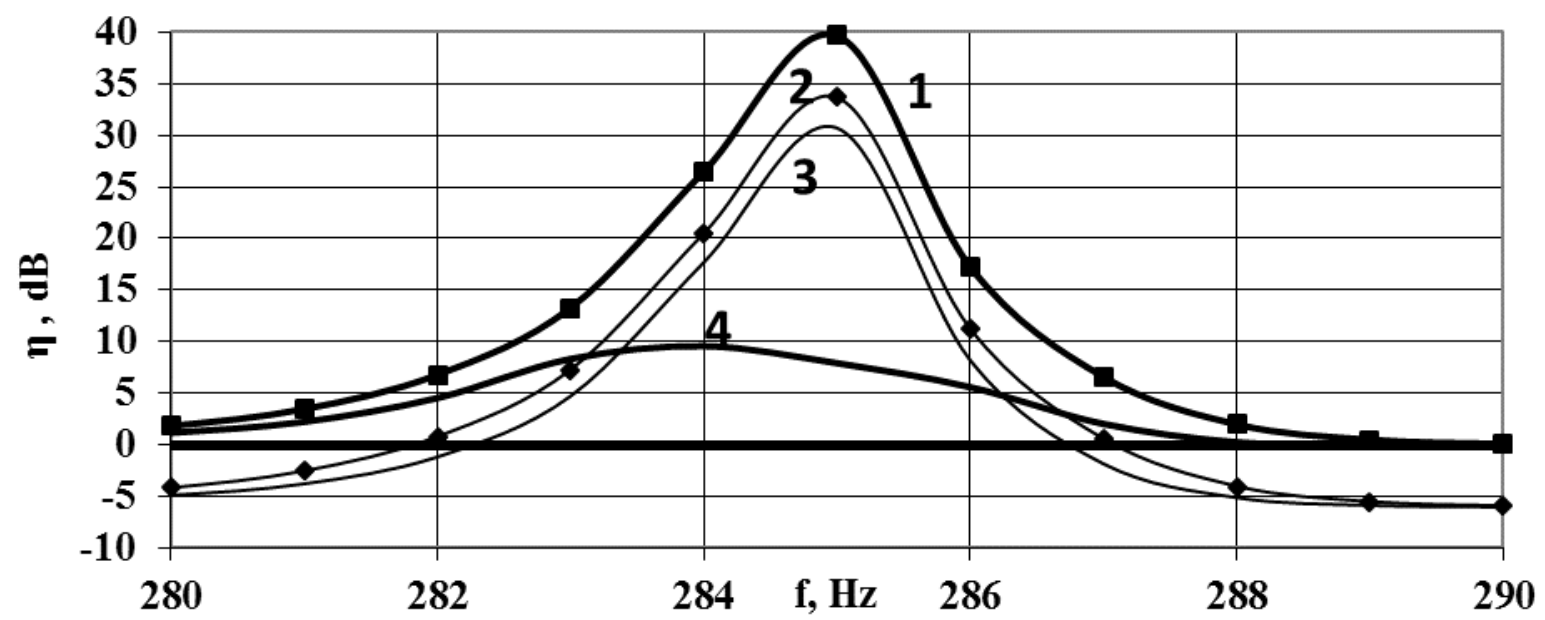

Figure 4:. Calculated efficiency by force sensors and by foundation of AVS in direction $\mathrm{z}$ with a resonant element fres $=285 \mathrm{~Hz}$. 1 - by the foundation, three AVS channels work, 2 - by the sensors, three AVS , 3 - by the sensors one AVS z , 4 - by the foundation one AVS z .

\section{CONCLUSION}

1. Vibration forces measured by a force sensor can markedly differ from those acting on the attached structures, when the sensor is exposed to the three-component force and when the sensor has transverse sensitivities. The error in measuring the force by this sensor 
depends on the magnitude of the transverse sensitivity coefficients and the ratio of forces acting on the sensor in three directions.

2. The efficiency of the AVS, determined by the results of force measurements by force sensors, can significantly differ from the effectiveness of its impact on the attached structures. 3. In mechanical systems with three-component vibrations to reduce the vibration forces transmitted to the attached structures, when force sensors, installed in the feedback loops, have the transverse sensitivities, the AVS use in three directions can several times and more increase the efficiency of active damping.

\section{ACKNOWLEDGEMENT}

The paper was written on account of the Russian Science Foundation (the RSF) Grant. Agreement No. 16-19-10292 dated May 12, 2016 between the Russian Science Foundation and Kaluga State University named after K.E. Tsiolkovsky.

\section{REFERENCES}

1. M.D. Genkin, V.G. Elezov, V.V. Yablonsky. Methods of active vibration damping, in collected articles: Dynamics and Acoustics of Machines. Moscow: Nauka, 1971, 70 - 88.

2. M.D. Genkin, V.G. Elezov, V.V. Yablonsky. Methods of machine controlled vibration protection. Moscow: Nauka, 1985, p. 240.

3. A.V. Kiryukhin, V.A. Tikhonov, A.G. Chistyakov, V.V. Yablonsky. Active vibration protection - purpose, principles, condition. 1. The purpose and principles of development. Problems of mechanical engineering and automation, Vol. 2, 2011, pp. 108-111.

4. A.V. Kiryukhin, O.O. Milman, A.V. Ptakhin, L.N. Serezhkin. Reduction of Vibration Forces and Pressure Pulsations in the Pipeline Compensators with Passive and Active Methods. International Journal of Mechanical Engineering and Technology, Vol. 9(9), 2018, pp. 1452-1462.

5. K.A. Pupkov, N.D. Egupov, I.G. Vladimirov, Yu.P. Kornyushin. Methods of the classical and modern theory of automatic control. Volume 3: Synthesis of automatic control system regulators. Moscow, MGTU im. N. E. Baumana Publ., 2004, p. 616. 